\title{
Plasticity of Expression of a Synaptic Vesicle Antigen in Adult Rat Superior Cervical Ganglion
}

\author{
Karen F. Greif \\ Department of Biology, Bryn Mawr College, Bryn Mawr, Pennsylvania 19010
}

\begin{abstract}
The effects of deafferentation and alterations of synaptic activity on levels of a synaptic vesicle-specific membrane protein (SV) were studied in the adult rat superior cervical ganglion (SCG) in vivo, using a monoclonal antibody directed against the protein. Levels of SV were quantified by radioimmunoassay. Deafferentation of the SCG results in a transient increase in SV levels in the SCG on days 7 and 10 after surgery, with levels then dropping below control levels on days 14, 21, and 30 after surgery. Immunohistochemical labeling of deafferented ganglia indicates that the increase is confined to the perikarya of principal ganglionic neurons. Levels of SV in an SCG target tissue, the iris, do not differ from control levels on day 7 after deafferentation, but are elevated at days 10, 14, and 30 after surgery. After reinnervation of the SCG, levels of $S V$ in the SCG are elevated above control values, but do not differ from control values in the iris. Treatment with chlorisondamine, which blocks synaptic transmission in sympathetic ganglia, produces a significant increase in SV levels in the SCG after $7 \mathrm{~d}$ of treatment. Long-term chlorisondamine treatment results in reductions in SV in the SCG after 14 and $28 \mathrm{~d}$. Treatment with phenoxybenzamine for $6 \mathrm{~d}$, which reflexly increases synaptic activity, produces a marked decrease in SV in the SCG. These results suggest that activity, mediated by transsynaptic factors, contributes to the regulation of synthesis of a synaptic vesicle protein in the SCG. The results further suggest that accumulation of synaptic vesicles in terminals of the principal ganglion neurons may help regulate the maintenance of normal synaptic vesicle pools within sympathetic ncurons.
\end{abstract}

While ample evidence for neural plasticity exists in the developing nervous system, studies of responses in adult systems have in general shown considerable limitations in the ability to respond to and recover from alterations in connectivity and activity. Among peripheral systems, the adult rat superior cervical ganglion (SCG) is one in which several studies have documented plasticity in response to experimental manipulations. Deafferentation of the SCG by section of the cervical sympathetic trunk (CST) in adult animals has been reported to produce only limited changes in ganglion size in the absence of regeneration of the CST (Gabella, 1976). If the CST is permitted to reinnervate the SCG, recovery of normal numbers of synapses occurs, as assessed by morphometric analysis, and restoration of normal

Received Feb. 21, 1986; revised May 27, 1986; accepted June 20, 1986

Chlorisondamine was generously donated by CIBA-Geigy Corporation. Phenoxybenzamine was a gift from Smith Kline and French Laboratories. Graduate students H. Trenchard and S. J. Carlsen participated in portions of this study. I thank Dr. J. Adler for helpful discussions and Dr. A. Smolen for discussions and criticism of earlier drafts of this paper. This research was supported by grants from the Dysautonomia Foundation, Inc. and from Bryn Mawr College. Additional support was provided by NSF Crrant PCM 84-00267.

Correspondence should be addressed to Karen F. Greif, Ph.D., at the above address.

Copyright (c) 1986 Society for Neuroscience $0270-6474 / 86 / 123628-06 \$ 02.00 / 0$ levels of the neurotransmitter synthetic enzymes, choline acetyltransferase and tyrosine hydroxylase (T-OH) are observed (Gabella, 1976; Raisman et al., 1974). Thus, the SCG is apparently capable of functional recovery from deafferentation. In contrast to these studies, recent work has shown that the biochemical nature of the ganglion can be significantly altered by deafferentation or pharmacological alteration of neuronal activity. Substance $P$ (SP), which is present in very low levels in normally innervated ganglia, rises significantly after deafferentation, treatment with chlorisondamine, which blocks cholinergic transmission in the SCG, or removal to explant culture (Adler and Black, 1984; Kessler and Black, 1982; Kessler et al., 1983). T-OH levels are increased by preganglionic electrical stimulation (Zigmond et al., 1980) and by treatment with reserpine (Thoenen et al., 1969).

The present study was undertaken in response to the observation that $7 \mathrm{~d}$ after deafferentation, levels of a synaptic vesicle antigen increase significantly in the SCG, despite the degeneration of presynaptic terminals within the ganglion (Greif, 1985). This observation stimulated the investigation of the regulation of numbers of synaptic vesicles in the adult rat SCG and in target tissue. Given that regeneration of the CST is possible, it is of interest to investigate whether the numbers of synaptic vesicles also recover to normal levels. In addition, since deafferentation not only removes presynaptic input to the SCG but reduces synaptic transmission, the synthesis of synaptic vesicles may be influenced by activity-related transsynaptic factors. A monoclonal antibody directed against a $65 \mathrm{kDa}$ integral membrane protein specific to synaptic vesicles (Matthew et al., 1981) has been used to indirectly monitor changes in vesicle number. Previous studies with this antigen in the developing rat SCG showed that the antigen undergoes significant postnatal increases that are correlated with the time course of synapse formation within the SCG and the accumulation of vesicles within the cytoplasm of principal ganglion cells (Greif and Reichardt, 1982). I sought to determine whether deafferentation or alterations of synaptic activity resulted in changes in levels of the vesicle antigen in the SCG and in the iris, and how such changes might be correlated.

\section{Materials and Methods}

\section{Characterization of antigen}

Monoclonal antibodies (SV 48) directed against a $65 \mathrm{kDa}$ integral membrane protein (SV) associated with the external surface of synaptic vesicles of all neurotransmitter types investigated thus far (Floor and Leeman, 1983; Matthew et al., 1981; Pfeffer and Kelly, 1985) were obtained from Drs. L. F. Reichardt and W. D. Matthew. The antigen can be localized to both presynaptic terminals and principal cell cytoplasm in the SCG (Greif and Reichardt, 1982). The antibody is used to indirectly monitor numbers of synaptic vesicles by measuring antigen levels after different experimental manipulations. While no direct conversion between SV levels and numbers of vesicles is possible at present, the approach has been used effectively in other systems (cf. Greif and Kelly, 
1983; Greif and Reichardt, 1982; Matthew et al., 1981; Pfeffer and Kelly, 1985).

\section{Surgery}

Surgery on adult female Sprague-Dawley rats was carried out using chloral hydrate $(35 \mathrm{mg} / \mathrm{kg}, 7 \%$ solution, i.p.) as general anesthetic. Deafferentation of the SCG was carried out by section of the CST 2-3 mm from the base of the ganglion. Rats were anesthesized, and the skin over the throat opencd and lightly retracted. The SCG was visualized by blunt dissection down to the carotid artery, with the aid of a surgical dissecting microscope (Aus Jena). The CST was cut using jeweler's forceps. In cases when reinnervation was not desired, a section of the CST was removed. To encourage reinnervation, the trunk was repeatedly crushed with jeweler's forceps, leaving a small fraction of the sheath intact. All surgery was carried out bilaterally to control for possible compensatory responses in the contralateral ganglion (cf. Dornay et al., 1985). The fascia and skin were sutured separately using 4-0 silk. All animals were given prophylactic doses of Bicillin $(100,000$ units) to prevent respiratory infections. Mortality was less than $1 \%$ with antibiotic treatment. Success of surgical procedures was assessed by the presence of marked ptosis following section of the CST. Animals were permitted to live for $4,7,10,14,21$, or $30 \mathrm{~d}$ following deafferentation to determine the time course of changes in SV. For studies of reinnervated ganglia, animals lived a minimum of $60 \mathrm{~d}$ after surgery. No ptosis was present in animals in which successful reinnervation had occurred by this time. At the time of death, careful visual inspection of the SCG was carried out to confirm the presence or absence of innervating fibers. Unoperated controls were aged-matched in all cases. Irides were dissected from experimental and control groups and processed in parallel to SCG samples.

Implantation of osmotic pumps (Alza; flow rate, $2.5 \mu \mathrm{l} / \mathrm{hr}$ ) was carried out under chloroform anesthesia. A small incision was made in the skin on the back and a subcutaneous pocket was prepared. The sterile, equilibrated pump was inserted and the incision was closed with wound clips.

\section{Drug treatment}

Blockade of ganglionic presynaptic activity was achieved using chlorisondamine (Ecolid Chloride, CIBA; $10 \mathrm{mg} / \mathrm{kg}$, s.c.) in physiological saline. Chlorisondamine competitively inhibits the binding of $\mathrm{ACh}$ to postsynaptic receptors. Rats were injected every $12 \mathrm{hr}$ for $7 \mathrm{~d}$ and immediately sacrificed. Synaptic blockade was confirmed by the presence of ptosis (cf. Kessler and Black, 1982). For long-term blockade of synaptic activity, rats were implanted with osmotic pumps (Alza) which were filled with a $5 \mathrm{mg} / \mathrm{ml}$ solution of chlorisondamine in Ringer's solution. This dose, selected in pilot studies to produce ptosis but permit long-term survival, was equivalent to an injected dose of $1.2 \mathrm{mg} / \mathrm{kg} / \mathrm{d}$. Mortality was $20 \%$ for $28 \mathrm{~d}$ survival. At higher doses, mortality rates were excessively high $(>50 \%)$. Control animals were implanted with pumps containing vehicle. All control animals survived for $28 \mathrm{~d}$. For reflex increases in synaptic activity, the alpha-adrenergic blocker phenoxybenzamine (SK\&F 688-A; $15 \mathrm{mg} / \mathrm{kg}$, i.p.) in DMSO was injected twice daily for $6 \mathrm{~d}$. The experiment was terminated $1 \mathrm{~d}$ early because of excessive drug toxicity. Control animals were treated with injections of vehicle.

\section{Quantitation of antigen levels}

The assay employed is a measure of the amount of whole tissue homogenate required to inhibit $50 \%$ of antibody binding in a standard solid-phase radioimmunoassay (RIA) (Greif and Reichardt, 1982). Ganglia from 4-6 adult rats were pooled to provide sufficient tissue for assay of each experimental condition at least twice. Tissue was homogenized

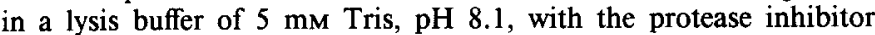
phenyl methyl sulfonylfuoride (PMSF) added. After $30 \mathrm{~min}$ incubation at $4^{\circ} \mathrm{C}$, tissue samples were stored in PBS and $10 \%$ sucrose. Protein determinations were by Amido-Schwarz assay, using BSA as a standard. Tissue samples were adjusted to $2-4 \mathrm{mg} / \mathrm{ml}$ initial protein concentration prior to preparation of a dilution series over $4 \mathrm{log}$ units. Samples of each tissue dilution were incubated with aliquots of monoclonal antibody at a dilution selected to be limiting in the solid-phase RIA (Klinman, 1972). Bound antibody was precipitated by spins at $100,000 \times g$ in an Airfuge. Antibody remaining in the supernatant was assayed by solid-phase RIA, using crude rat brain synaptosomes as the antigen (Jones and Matus, 1974); 50\% inhibition points (I-50) were determined graphically and standardized to an initial protein concentration of 4 $\mathrm{mg} / \mathrm{ml}$. Additional corrections for differences in ganglionic protein content were calculated according to (I-50 experimental) $\times$ [control ganglion ( $\mathrm{mg}$ protein)/experimental ganglion (mg protein)]. For assay of target tissue, irides from experimental and control animals were processed similarly.

\section{Localization of antigen}

Ganglia to be used for immunohistochemistry were removed, desheathed, and immediately mounted on cryostat chucks using TissueTek. At least 2 ganglia from different animals in each experimental condition were used. Samples were frozen with dichlorodifluoromethane (Histofreeze). Frozen unfixed sections of SCG, $8 \mu \mathrm{m}$ thick, were cut and either used immediately or stored desiccated at $-80^{\circ} \mathrm{C}$. Affinitypuritied HRP-conjugated anti-mouse immunoglobulin antisera (Sigma) were preabsorbed with rat liver acetone powders. Sections were incubated with blocking serum for $1 \mathrm{hr}$, then overnight with SV 48 at $4^{\circ} \mathrm{C}$, followed by washing with PBS-BSA $(1 \mathrm{mg} / \mathrm{ml})$. Sections were then incubated with HRP-conjugated second antibodies for 2-4 hr at room temperature. Following final washes in PBS, label was visualized using $3,3^{\prime}$-diaminobenzidine and $0.01 \% \mathrm{H}_{2} \mathrm{O}_{2}$ in $50 \mathrm{~mm}$ Tris, $\mathrm{pH} 7.6$, for 15 $30 \mathrm{~min}$. Sections were mounted in $60 \%$ glycerol in PBS and viewed using a Zeiss Universal microscope equipped with Nomarski optics. Experimental and control tissues were processed in parallel for immunocytochemistry. Controls included replacement of primary antibody with BSA or normal mouse serum. Higher backgrounds were consistently observed using normal serum.

\section{Results}

Deafferentation of adult SCG produced, on average, a $24 \%$ reduction in total protein content per ganglion (control SCG: $84.4 \pm$ $2.5 \mu \mathrm{g}$ protein per ganglion; deafferented SCG: $64.1 \pm 6.4 \mu \mathrm{g}$ ). This reduction was observed in samples beginning on day 7 after surgery. No reduction in protein content was observed in ganglia taken $4 \mathrm{~d}$ after surgery. No significant changes in protein content in the iris were observed following deafferentation of the SCG (iris: $39.6 \pm 1.1 \mu \mathrm{g}$ protein). Treatment with chlorisondamine, phenoxybenzamine, saline, and DMSO did not significantly alter protein content of either the SCG or iris.

Adult rats were killed 4, 7, 10, 14, 21, and $30 \mathrm{~d}$ after section of the CST to determine the time course of changes in SV levels within the ganglion. The results of assays of SV in SCG tissue samples at varying times after bilateral deafferentation of the SCG are shown in Figure 1. Data are presented as the relative specific activity (R.S.A.) of experimental groups as compared to control tissue. I-50 levels were normalized for ganglionic protein content to permit direct comparison of experimental and control tissues. An equal SV content would be expressed as a R.S.A. value of 1.0. Four days after deafferentation, levels of SV do not differ from control values. However, by day $7, \mathrm{SV}$ levels are 1.51 times greater than control values ( $t$ test; $p<$ $0.005)$ and elevated SV levels persist at day 10 (R.S.A. = 1.46). This increase in SV level is transient, with values dropping to 0.68 of control values by $14 \mathrm{~d}$ after surgery, 0.59 by $21 \mathrm{~d}$, and 0.40 by $30 \mathrm{~d}(t$ tests; $p<0.05)$. Levels of SV in the reinnervated SCG are elevated at 1.35 ( $t$ test; $p<0.025$ ).

Increases in SV levels observed in the SCG are not the result of nonspecific interactions between SV 48 and ganglionic tissue, since RIA of other neuron-specific antigens indicates no significant alterations in antigen levels (Table 1; cf. Greif and Trenchard, 1984).

Histological analysis of sections of SCG stained for SV reveals that most punctate terminal stain associated with presynaptic terminals (Fig. 2A) (cf. Greif and Reichardt, 1982) has disappeared by $7 \mathrm{~d}$ after deafferentation, suggesting that the increase in SV levels cannot be due to the persistence of antigen in presynaptic terminals (Fig. $2 B$ ). No evidence for punctate terminal labeling can be observed in sections from $30 \mathrm{~d}$ deafferented ganglia (Fig. $2 C$ ). At the same time, the perikarya of the principal ganglionic neurons are heavily labeled. These obser- 


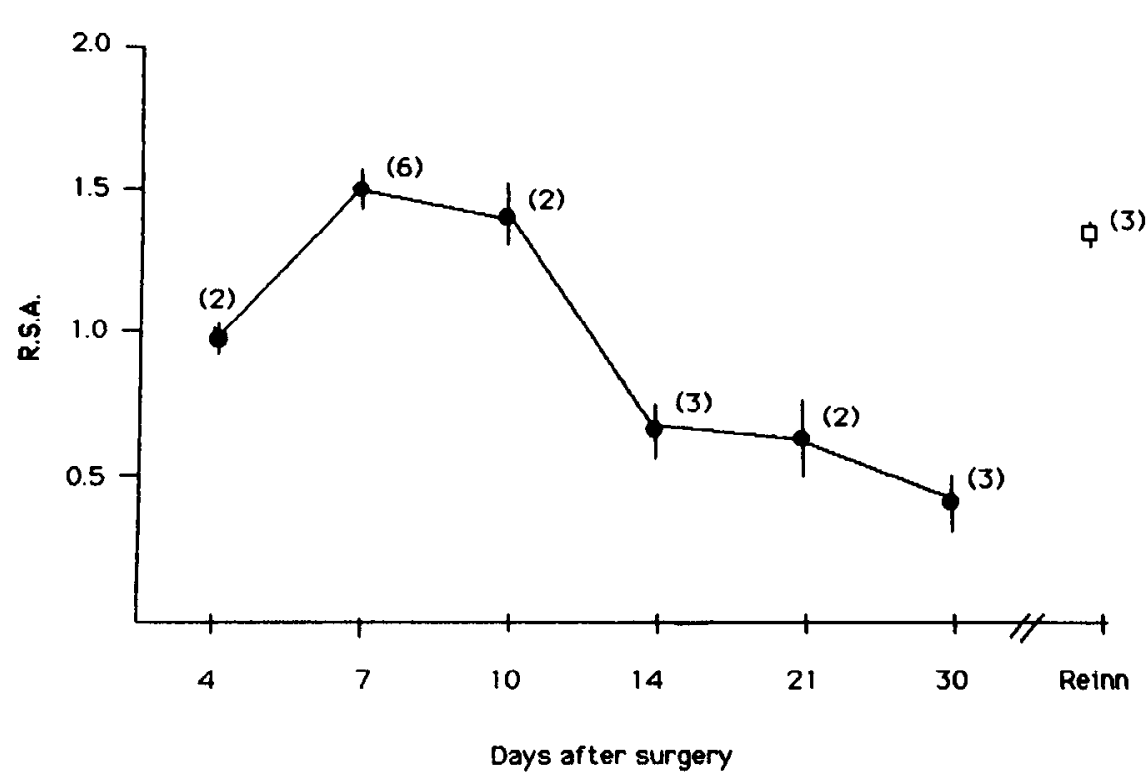

Figure 1. Time course of changes in SV in the SCG after bilateral deafferentation of adult rat SCG. Surgery was carried out on day 0 , with rats killed on the days indicated. $R . S . A .=\mathrm{I}-50$ experimental/I-50 aged-matched controls. I-50s were normalized for ganglionic protein content. Bars, 1 SEM. Numbers in parentheses indicate numbers of assays. A significant increase in SV is observed in deafferented SCG on days 7 and 10 after surgery. SV is significantly less than control values at 14,21 , and $30 \mathrm{~d}$ nervated SCG ( $>60 \mathrm{~d}$ after surgery). R.S.A. is significantly greater than control values. See text for details. after surgery. Open square, the rein-

vations strongly suggest that increases in SV levels within the deafferented SCG must occur within the principal ganglion neurons. No histological evidence for SV accumulation or production in other cell types of the SCG was obtained. Immunocytochemical labeling of sections of reinnervated SCG shows the reappearance of punctate terminal staining between the principal ganglionic neuron perikarya (Fig. 2D).

Assays of SV levels in the iris, a primary target of the SCG, were carried out in order to determine whether the increases in SV observed in the SCG following deafferentation might be the result of blocked axoplasmic transport. If this were the case, decreased levels of transported SV might be observed in target tissue. Results of assays of SV in iris are shown in Figure 3. SV levels in the iris 4 and $7 \mathrm{~d}$ after deafferentation of the SCG do not differ significantly from control values ( $t$ test; $p>0.10$ ). However, at longer survival times, levels of SV in iris were significantly increased above control levels, beginning at day 10 after surgery (R.S.A. $=1.56, t$ test; $p<0.05$ ) and remaining elevated at 1.63 and 1.42 times control values at 14 and $30 \mathrm{~d}$, respectively ( $t$ tests; $p<0.05$ ). A small increase in SV in the iris was observed at $21 \mathrm{~d}($ R.S.A. $=1.10)$, but was not statistically significant. These findings suggest that the increase observed in $\mathrm{SV}$ in the SCG at $7 \mathrm{~d}$ cannot be the result of altered axoplasmic transport.

The increase in SV levels $7 \mathrm{~d}$ after deafferentation can also be produced by $7 \mathrm{~d}$ of treatment with chlorisondamine, which blocks cholinergic synaptic transmission in the SCG. As is shown in Figure 4, treatment with chlorisondamine for $7 \mathrm{~d}$ results in an R.S.A. of 1.89 ( $t$ test; p $<0.005$ ). This increase occurs in the presence of an intact CST, which suggests that activity plays a critical role in mediating the increase in SV. Immunohistochemical staining of sections of the SCG yields results that do

Table 1. RIA of neuronal antigens in $7 \mathrm{~d}$ deafferented SCG

\begin{tabular}{lll} 
Antibody & Antigen & RSA ( \pm 1 SEM) \\
\hline 48 & SV & $1.51 \pm 0.06^{*}$ \\
$22^{a}$ & HeS-PG (core protein) & $1.18 \pm 0.06^{* *}$ \\
$3^{a}$ & HeS-PG (side chain) & $0.83 \pm 0.10^{* *}$ \\
\hline
\end{tabular}

${ }^{a}$ Data taken from Greif and Trenchard (1984).

$* p<0.005$.

$* * p>0.10$. not differ markedly from those of control ganglia (data not shown). Longer-term treatment with chlorisondamine yields results similar to that observed with longer survival periods after deafferentation. Levels of SV in the SCG drop to 0.98 times control values after $14 \mathrm{~d}$ of treatment, and to 0.62 times control after $28 \mathrm{~d}$. Treatment with phenoxybenzamine, which increases synaptic activity, produces a marked reduction in SV in the SCG (R.S.A. $=0.50, t$ test; $p<0.05$ ). No significant differences between vehicle-treated and normal adult rat SCGs were observed.

Treatment with chlorisondamine for $7 \mathrm{~d}$ leads to an increase in SV levels in the iris, but this increase is not statistically significant ( $t$ test; $p>0.10$ ) (Fig. 4). However, long-term chlorisondamine treatment results in significant increases in SV content in the iris ( $t$ tests; $p<0.05$ ). Treatment with phenoxybenzamine for $6 \mathrm{~d}$ produces a significant decrease in SV levels in the iris (R.S.A. $=0.44, t$ test; $p<0.025$ ). When reinnervation of the SCG is permitted, levels of SV in the iris return to control values, suggesting that alterations in levels of SV in a SCG target may also be the result of altered activity levels within the ganglion.

\section{Discussion}

The results from assays of deafferented SCGs from adult rats indicate that levels of SV undergo rapid changes following surgery. A significant increase in SV levels was observed $7 \mathrm{~d}$ after section of the CST, followed by a fairly rapid decline in antigen level. The present study has not resolved finely the time course of changes in antigen levels; nevertheless, the apparent induction in SV occurs within $7 \mathrm{~d}$ after surgery. The pattern of changes is unexpected; it is reasonable to predict that levels of a synaptic vesicle antigen would drop with the degeneration of presynaptic terminals. This is indeed the case for Synapsin 1, a synaptic vesicle-specific phosphoprotein, where the existence of a pool of approximately $40 \%$ of total SCG Synapsin 1 was demonstrated in neuronal perikarya $1-4$ weeks after removal of preganglionic input in the rabbit SCG (Nestler and Greengard, 1982). This finding is in agreement with the results of the present study, where $40 \%$ of control levels of SV in the SCG were observed $30 \mathrm{~d}$ following deafferentation, which suggests that Nestler and Greengard (1982) studied antigen levels after the transient peak reported in the present study. Alternatively, the rabbit may not exhibit the same plasticity as the rat. The increase in SV levels is consistent with a report of increased immuno- 

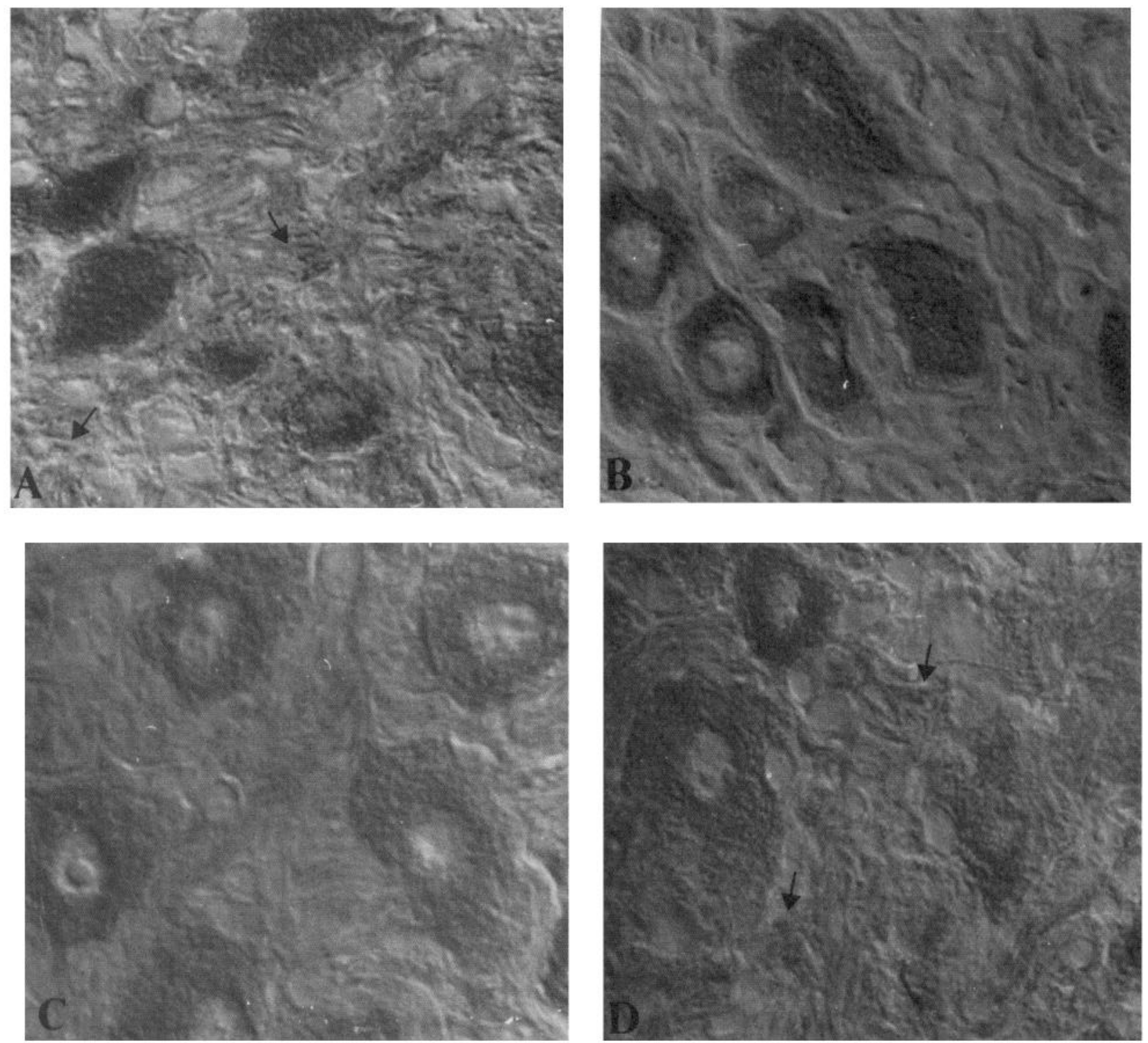

Figure 2. Immunohistochemical labeling of SCG with SV 48. Distribution of SV in $8 \mu \mathrm{m}$ frozen sections of unfixed SCG was visualized using HRP-conjugated second antibodies. Nomarski optics. 1250×.A, Unoperated SCG (control). Heavy staining of principal ganglion neuron cytoplasm and staining of presynaptic terminals (arrows) between cell bodies is shown. B, Deafferented SCG $7 \mathrm{~d}$ after surgery. Most punctate terminal stain has disappeared. Heavy staining of cell cytoplasm is present. $C$, Deafferented SCG $30 \mathrm{~d}$ after surgery. No evidence for punctate terminal stain is present. In addition, there is a suggestion of some loss of label from cell bodies. $D$, Reinnervated SCG $70 \mathrm{~d}$ after surgery. Punctate terminal stain has reappeared (arrows). See text for discussion.

fluorescence labeling in deafferented SCG, using a polyclonal antisera directed against synaptic vesicles (Hooper et al., 1980). However, the investigators did not specify the time after deafferentation that sections were prepared.

The increase in SV levels following deafferentation must be the result of an accumulation of SV in neuronal cell perikarya, since immunohistochemical labeling of SV in deafferented ganglia confirms the disappearance of punctate staining associated with presynaptic terminals (cf. Greif and Reichardt, 1982). Staining of non-neuronal cells in the SCG was not observed following deafferentation. The increase in SV within cell bodies is not visible in histological sections, probably as a result of the limited quantitative sensitivity of the method.

What factors might contribute to the increase in SV in the SCG after deafferentation? One immediate concern is that deafferentation leads to blocked or slowed axoplasmic transport of
SV to terminals in target tissue. If this were the case, SV would accumulate in the SCG and levels of SV might be expected to drop in targets, even in the absence of neuronal activity, because of protein turnover. Although the turnover rate of SV is not known, the present results argue against blocked axoplasmic transport as the mechanism responsible for the observed increase of ganglionic SV. Assay of SV levels in the iris after deafferentation did not reveal any decrease in antigen; SV levels showed a tendency to increase in target tissue with longer survival times. Given that this is the case, it seems likely that the increase in SV levels represents new synthesis of SV by ganglionic neurons. Studies of the role of new protein synthesis in mediating SV increases are anticipated.

Deafferentation of the SCG results in the cessation of normal synaptic activity within the SCG. If levels of activity are either directly or indirectly regulating ganglionic SV levels, then agents 
Figure 3. Time course of changes in $S V$ in iris after deafferentation of the SCG. Data presented as in Figure 1. No differences in SV levels are observed 4 and $7 \mathrm{~d}$ after surgery. SV levels in iris at 10,14, and 30 days after surgery are significantly above control values. Open square represents iris in reinnervated SCG animals. SV levels do not differ from control levels. See text for discussion.

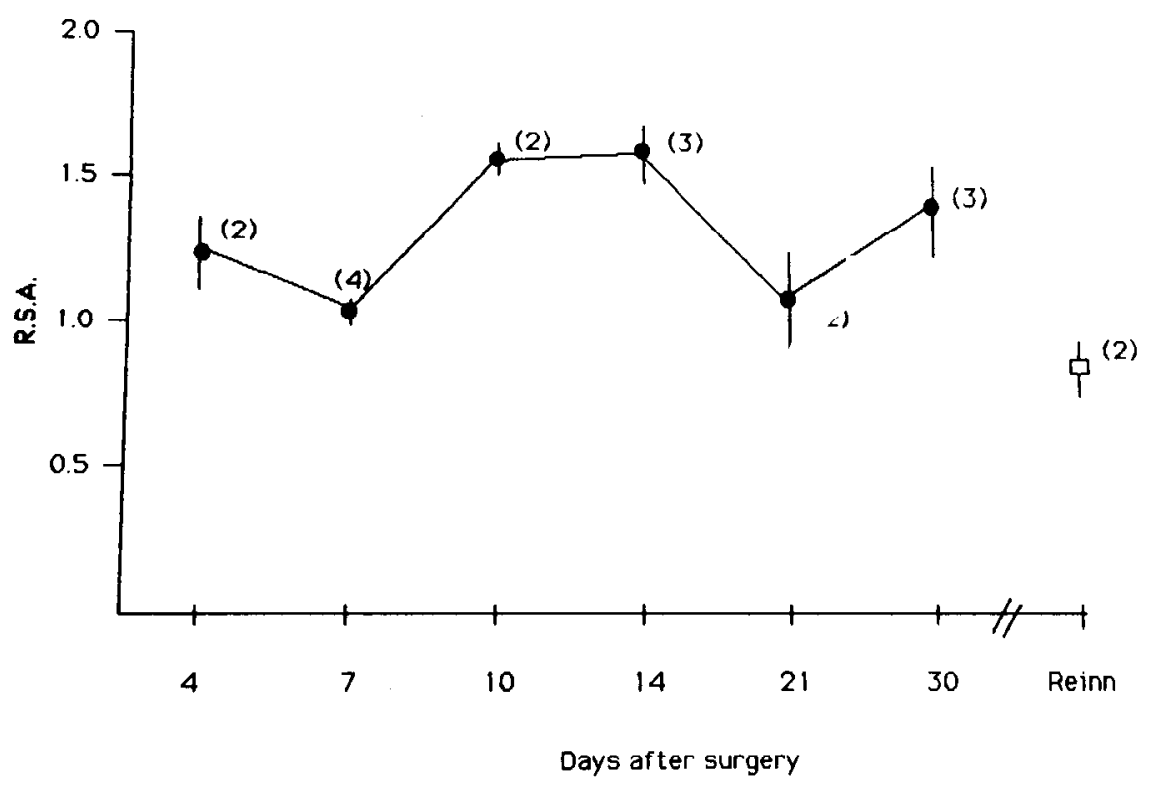

that alter synaptic activity might be expected also to produce changes in SV levels. Treatment of rats with the ganglionic blocking agent, chlorisondamine, for $7 \mathrm{~d}$ produces an increase in SV levels within the SCG. It is possible that a portion of this increase is due to changes in SV levels in intact presynaptic terminals. However, because of the similarities in response to deafferentation and ganglionic blockade, it is likely that significant changes are occurring in principal ganglionic neurons. Longer term ganglionic blockade results in reductions in SV in the SCG, similar to those observed following deafferentation. Treatment with phenoxybenzamine, which reflexly increases presynaptic activity (cf. Kessler and Black, 1982) produces a marked decrease in SV levels in both SCG and iris. It is not possible on the basis of this study to determine whether altered levels of SV are confined to cell cytoplasm or also occur in presynaptic terminals.

The above findings are consistent with the hypothesis that activity plays a critical role in regulating expression of neuronal proteins. At present, it is not possible to rule out the possibility that other factors, such as those produced by degenerating nerve terminals, may contribute to the induction of SV after deafferentation. The results of long-term treatment with chlorisondamine suggest that altered activity plays the primary role in regulating SV levels after deafferentation.

Previous studies have demonstrated that deafferentation of adult ganglia blocks the normal induction of T-OH in the SCG following catecholamine depletion produced by treatment with reserpine (Kornack et al., 1985; Raisman et al., 1974; Thoenen et al., 1969). T-OH levels also increase following preganglionic nerve stimulation (Zigmond et al., 1980), consistent with increased release of catecholamines at ganglionic targets. Alterations in activity are known to induce changes in the expression of SP within the SCG (Kessler and Black, 1982; Kessler et al., 1981, 1983; Kornack et al., 1985). Agents that decrease neuronal
Figure 4. Effects of pharmacological treatments on SV levels in SCG and iris. Rats were treated with chlorisondamine (10 mg/kg, s.c.; dark shading) for $7 \mathrm{~d}$, or with phenoxybenzamine $(15 \mathrm{mg} / \mathrm{kg}, \mathrm{i} . \mathrm{p}$;; light hatching) for 6 $\mathrm{d}(P 6)$. Treatment with chlorisondamine for 14 and $28 \mathrm{~d}$ (medium and light shading) was via osmotic pumps (equivalent dose, $1.2 \mathrm{mg} / \mathrm{kg} / \mathrm{d}$ ). White bars are pooled control (vehicle-injectcd) animals. Data presented as in Figure 1, with control values standardized to a value of 1.0 . Treatment with chlorisondamine significantly increases SV levels in the SCG at $7 \mathrm{~d}$. The increase in iris is not statistically significant. Levels of SV in SCG after $28 \mathrm{~d}$ are significantly less than control values. Increases in iris at both 14 and $28 \mathrm{~d}$ are significant. Treatment with phenoxybenzamine significantly decreases SV levels in both SCG and iris. See text for further discussion.

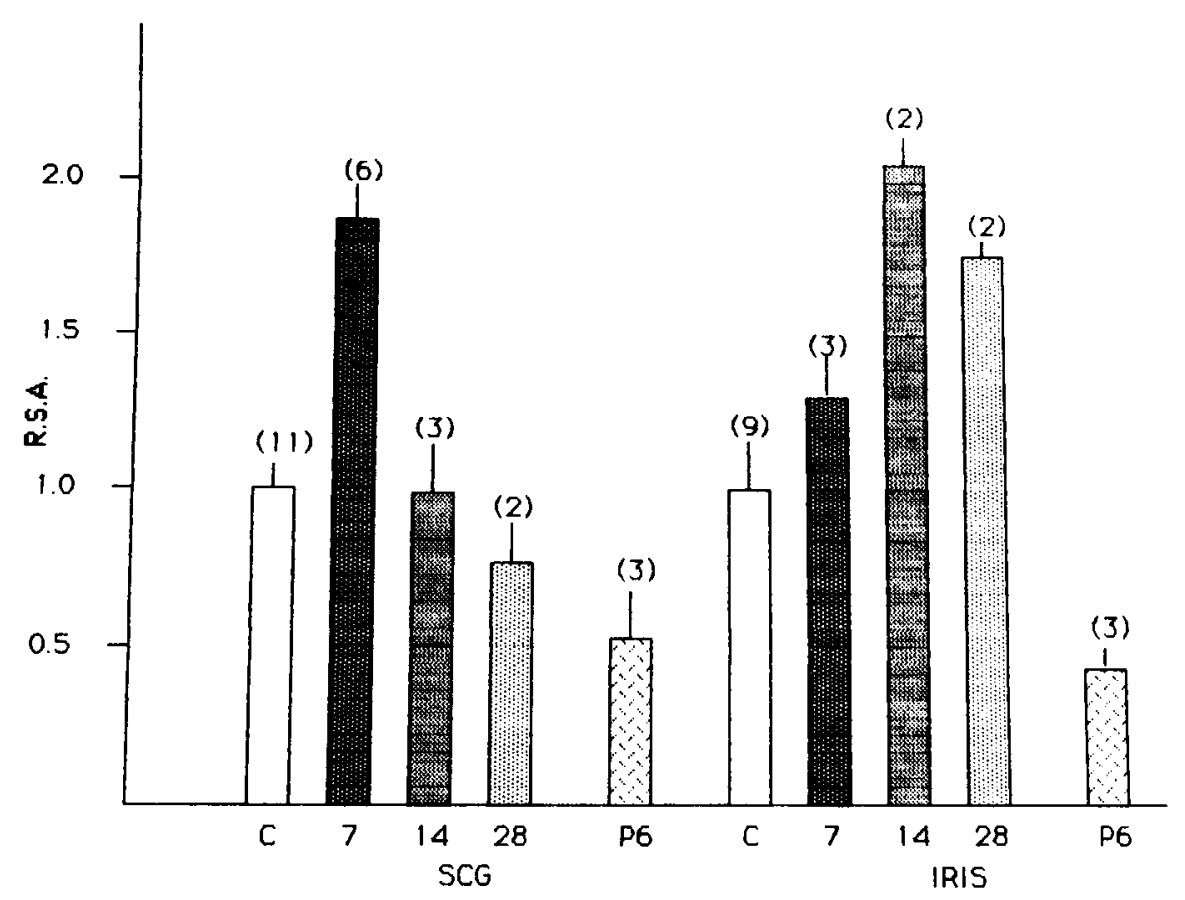


activity tend to increase synthesis of SP; stimulation of the SCG blocks these increases (cf. Kessler and Black, 1982; Kessler et al., 1981, 1983). However, whether increased levels of SP persist after long-term deafferentation was not examined. Treatment with phenoxybenzamine, which increases ganglionic activity, produces a decrease in ganglionic SP. The induction of SP in vitro is blocked by treatment with depolarizing agents such as veratridine or $\mathrm{K}^{+}$, suggesting that neuronal depolarization is involved (Adler and Black, 1984).

If reduced activity in the SCG plays a critical role in inducing increases in SV levels, what might contribute to the transient nature of the increase? Reinnervation studies indicate that SV levels are slightly elevated in the SCG after synaptic transmission is reestablished. However, SV levels do not remain elevated after survival periods of 14,21 , or $30 \mathrm{~d}$ after deafferentation. In the present study, levels of SV in a primary target tissue, the iris, show a slow increase after deafferentation. Elevated levels of SV are observed in the iris 14 and $30 \mathrm{~d}$ after deafferentation, at the same time that SV levels decrease significantly below control levels in neuron perikarya. It is possible that the accumulation of SV in the inactive terminals of ganglionic neurons might regulate continuing synthesis of SV in the neuronal cell body. As long as SV levels remain high in terminals, synthesis of SV by the principal ganglionic neurons might remain depressed. Thus, "end-product" regulation of SV synthesis may contribute to the overall pattern of SV response after deafferentation.

The results of the present study offer further evidence that considerable plasticity in response to changing neuronal environments exists in the sympathetic nervous system. The adult nervous system remains sensitive to alterations in synaptic activity levels and responds rapidly to such changes.

\section{References}

Adler, J. A., and I. B. Black (1984) Plasticity of substance P in mature and aged sympathetic neurons. Science 225: 1499-1500.

Dornay, M., V. H. Gilad, and G. M. Gilad (1985) Compensatory changes in contralateral sympathetic neurons of the superior cervical ganglion and in their terminals in the pineal gland following unilateral ganglionectomy. J. Neurosci. 5: 1522-1526.

Floor, E., and S. E. Leeman (1983) Synaptic vesicles containing substance $P$ bind a monoclonal antibody specific for synaptic vesicles. Soc. Neurosci. Abstr. 9: 1026.

Gabella, G. (1976) Structure of the Autonomic Nervous System, Chapman and Hall, London.

Greif, K. F. (1985) Plasticity of synaptic vesicle number in adult rat superior cervical ganglion following deafferentation and ganglionic blockade. Soc. Neurosci. Abstr. 11: 1103.
Greif, K. F., and A. S. Kelly (1983) Quantitation of synaptic vesiclc antigen in rabbit superior colliculus during normal development and after neonatal visual cortical lesion. Brain Res. 275: 143-147.

Greif, K. F., and L. F. Reichardt (1982) Appearance and distribution of neuronal cell surface and synaptic vesicle antigens in the developing rat superior cervical ganglion. J. Neurosci. 2: 843-852.

Greif, K. F., and H. I. Trenchard (1984) Evidence for transsynaptic regulation of neuronal cell surface heparan sulfate proteoglycan in developing rat superior cervical ganglion. J. Cell Biochem. 26: 127133.

Hooper, J. E., S. S. Carlson, and R. B. Kelly (1980) Antibodies to synaptic vesicles purified from Narcine electric organ bind a subclass of mammalian nerve terminals. J. Cell Biol. 87: 104-113.

Jones, D. H., and A. I. Matus (1974) Isolation of synaptic plasma membranes from brain by combined flotation-sedimentation density gradient centrifugation. Biochem. Biophys. Acta 356: 276-287.

Kessler, J. A., and I. B. Black (1982) Regulation of substance P in adult rat sympathetic ganglia. Brain Res. 234: 182-187.

Kessler, J. A., J. E. Adler, M. C. Bohn, and I. B. Black (1981) Substance $P$ in principal sympathetic neurons: regulation by impulse activity. Science 214: 335-336.

Kessler, J. A., W. O. Bell, and I. B. Black (1983) Substance P levels differ in sympathetic target organ terminals and ganglion perikarya. Brain Res. 258: 144-146.

Klinman, N. R. (1972) The mechanism of antigenic stimulation of primary and secondary clonal precurser cells. J. Exp. Med. 136: 241260.

Kornack, D. R., J. E. Adler, and I. B. Black (1985) Extracellular factors regulate neurotransmitter plasticity in mature sympathetic ncurons. Soc. Neurosci. Abstr. 11: 1142.

Matthew, W. D., L. Tsavaler, and L. F. Reichardt (1981) Identification of a synaptic vesicle specific membrane with a wide distribution in neuronal and neurosecretory tissue. J. Cell Biol. 91: 257-269.

Nestler, E. J., and P. Greengard (1982) Distribution of protein 1 and regulation of its state of phosphorylation in the rabbit superior cervical ganglion. J. Neurosci. 2: 1011-1023.

Pfeffer, S. R., and R. B. Kelly (1985) The subpopulation of brain coated vesicles that carries synaptic vesicles contains two unique polypeptides. Cell 40: 949-957.

Raisman, G., P. M. Field, A. J. C. Ostherg, I. L. Iversen, and R. E. Zigmond (1974) A quantitative ultrastructural and biochemical analysis of the process of reinnervation of the superior cervical ganglion in the adult rat. Brain Res. 171: 1-16.

Thoenen, H., R. A. Mueller, and J. Axelrod (1969) Increased tyrosine hydroxylase activity after drug induced alteration of sympathetic transmission. Nature 221: 1264.

Zigmond, R. E., A. Chalazonitis, and T. Joh (1980) Preganglionic nerve stimulation increases the amount of tyrosine hydroxylase in the rat superior cervical ganglion. Neurosci. Lett. 20:61-65. 